\section{Differential multipole method for microstructured optical fibers}

S. Campbell, R. C. McPhedran, and C. Martijn de Sterke

School of Physics, Gentre of Excellence for Uliruhigh-bandwidth Devices for Optical Systems, Liniversity of Sydney. Sydrey, New South Wales 2006. Australic

\section{C. Botten}

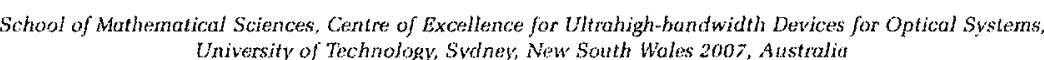

Received March 29, 2004; accepted June 28, 2004

We describe the differential nultipole method, an extended multipole method used to calculate the modes of

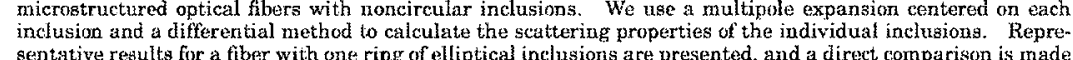
with an existing method. The new method is also applied to a microstruetured optical liber witti seven rings of elliptical inclusions, which is found, in effect, to support a single polarization of the fundamental roode. 2004 Optical Society of Aneriea
OCIS cosdes: $0610.2280,060.2400,060.4510$.

\section{INTRODUCTION}

Microstructured optical fibers, or MOFs, come in a wide

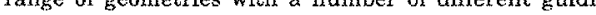
mechanisms. The majority of existing MOFs consist of an array of low-index inclusions arranged around a highindex core, where light is guided through a similar mechanism to the total internal reflection in convention fibers. Other MOF geometries include periodic arrange or air core, where confinement is due to coherent Bragg or air core,
scattering.

Modeling these fibers presents a number of challenges: They generally lack rotational symmetry, have large refractive-index contrasts, and require full vector models when high accuracy or vector properties, such as birefringence, are required. Predicting the confinement loss in MOF's has also presented significant challenges. The most widely used technique, the plane-wave method, defines the MOF in a supercell and imposes periodic bound ary conditions. Because of this, confinement loss cannot be calculated because the finite geometry has been replaced with an infinite on

Several methods that are capable of calculating both the propagation constants and the confinement losse have been reported in the literature. With beam propagation methods, the scalar or vector wave equation is used to propagate an input pulse along a fiber, from which the guided modes propagation constants can be determined. Although beam propagation methods are applithe method requires significant computational resourcer to achieve modest accuracy in los calculations. rection and a Fourier decomposition is used in the azimuthal direction. The method correcty determines the radiating of the modes for abitrary fiber so it can calculate accuracy can be achieved with this method.

In the multipole method $d^{\mathbb{3}, 6}$ each dielectric boundary in the system is treated as a source of radiating fields. In current implementations for circular inclusions, the method achieves high accuracy and rapid convergence with modest computational resources. Systems with large numbers of inclusions can be modeled, and for structures with discrete rotational symmetries the comput tional overhead is further reduced by exploitation of the symmetry properties of the modes. The method is limited to nonintersecting circular inclusions, and convergence problems arise as the spacing between the inclusions decreases

with noner we extend the multipole method to MOF with noncircular inclusions through use of a fast Fourie properties of the individual inclusions. Some accuracy and speed are sacrificed in pursuit of generality: however. it is still well suited to a desktop environment. The multipole method requires minimal reformulation for noncircular inclusions, and existing implementations of the method require the replacement of only a single routine. The new method is suitable for most needs, retaining the strengths of the multipole method with increased generality

In this paper we combine two existing techniques, so only the details sufficient to outline the differential multipole method (DMM) formulation are presented. Further details can be found in the original publications. ${ }^{5-6}$ After the formulation is introduced, the convergence of with the $\mathrm{ABC}$ methud for a fiber with six elliptical inclusions. The method is then applied to a MOF with seven rings of elliptical inclusions, which is seen to support, effectively, a single polarization of the fundamental mode. This fiber has similar dimensions to a recently fabricated istic design.

\section{FORMULATION}

Here the formulation of the DMM is presented. First the multipole method for MOFs is introduced to establish the context for the required extensions to noncircular inclusions. Because of the decoupling between the inclusions' position and shape in the multipole method, no assumptions are necessary in the formulation as to the inclusion cross-sectional geometry, which enters through the reflec tion matrix $\mathbf{R}$. The reflection matrix relates the incident and scattered felds for an inclusion. For circular inclusions this med fix for an inclion. requires a numerical integration. The trut matrix qualitative calculation of the refection matrix is de scribed in Subsection 2.B, and the formulation of the numerical integration is described in Subsection $2 \mathrm{C}$. Unless defined otherwise, in this paper lower-case letters in bold represent vectors and capital letters in bold represent matries.

A. Multipole Formulation

The geometry consists of a finite number $N_{\mathrm{c}}$ of inclusion of refractive index $n_{l}$, where $l$ refers to the $l$ th inclusion, and all inclusions are oriented parallel to the $z$ axis an enbedded in a background material of refractive index $n_{e}$, The incle that ancely without intersecting any other escribed circle can be drawn, as shown in Fig. 1. For circular inclusions this reduces to the requirement for nonintersecting inclusion in the original formulation. Outside the microstruetured interior of the fiber the MOF is enclosed by a jacket material of possibly complex refractive index. Here the jacket is not included explicitly; however, we indicat where it enters the formulation.

All field components are taken to have an $\exp i\left(\beta_{2}\right.$ $\omega t)$ dependence. Here $\beta$ is the propagation constant of the mode and $\omega$ is the angular frequency of the mode, which is related to the free-space wave number by $=c k_{0}$. The effective index of a mode is defined as $n_{\mathrm{ef}}$ lated to the confinement loss in decibels per kilometer by

$$
\operatorname{loss}(\mathrm{dB} / \mathrm{km})=\frac{20}{\ln (10)} \frac{2 \pi}{\lambda} n_{\mathrm{elf}}^{i} \times 10^{9},
$$

where $\lambda$ is in micrometers. Specifying the $E_{z}$ and $H_{z}$ field components is sufficient to specify the fields in the Maxwell's index $n_{2}$ is constant, so the longitudinal fold compon $E_{2}$ and $H_{2}$ satisfy a the $E_{z}$ and $H_{z}$ satisfy a radial Helmholtz equation ( $V^{2}$ ground.

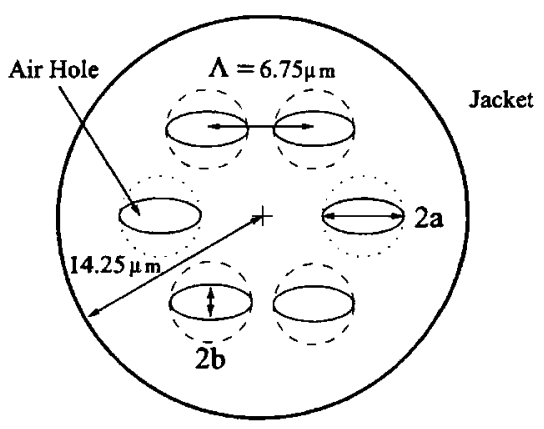

Fig. 1. Schematic of the MOF considered. $\Lambda$ is the center-to-
center separation, $a$ is the semimajor inclusion axis, and $b$ is the center separation, $a$ is the semimajor inclusion axis, and $b$ is the
semiminor inclusion axis, where $b=\eta \alpha$. The case of $\eta=1$ i semiminor inclusion axis, where $b=\eta \alpha$. The case of $\eta=11$
denoted by the dashed and dotted circles and defines the escribed circle for each inclusion. The difference
dotted circles is discussed in Section 3 .

Inside each inclusion the fields satisfy the same equation, with $\kappa \ldots k_{1}^{1} \ldots\left(k_{0}^{2} n_{i}^{2}-f^{2}\right)^{1 / 2}$. Local field expansions for $E_{2}$ and $H_{2}$ are made about the $l$ th inclusion in the coordinate system $\mathbf{r}_{t}=\left\{r_{l}, \theta_{t}\right\}$ in cylindrical harmonic functions, valid in an annuluz extending from the escribed circle of the
inclusion:

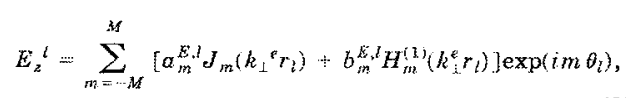

where $J_{m}(z)$ is the Bessel function of order $m, H_{m}^{(1)}(z)$ is the Hankel function of the first kind of order $m$, and a similar expression applies for the magnetic field. The expansions are truncated from $-M$ to $M$. The $J_{m}$ terms represent the regular incident part of the $E_{z}$ field for inpart of the field. At each inclusion, the reflection matrix connects the outgoing waves and the standing wave $\mathbf{b}^{t}=\mathbf{R}^{\prime} \mathbf{a}$, where $\mathbf{a}^{l}$ denotes the regular part of the fiel in the partitioned vector notation $\mathbf{a}^{l}$
$=\left[\mathbf{a}^{b, r^{T}} \mathbf{a}^{H, i, i^{r}}\right]^{T}, T^{T}$ denotes transposition, and $\mathbf{b}^{l}$ denotes the outgoing part of the field.

A global description of the fields was introduced by Wijngaard, ${ }^{11-1.3}$ who reasoned that the regular part of the field $\mathbf{a}$, in the annulus surrounding the $l$ th inclusion, is due to the outgoing contributions from all other inclusion $\{\mathbf{b}\}_{j+t}$ and a standing wave from the jacket, if presen

$$
E_{F}=\sum_{j \neq l}^{N_{c}} \sum_{m=-M}^{M} b_{m i}^{E \cdot j} H_{m}^{(h)}\left(k_{\perp}^{e} r_{r}\right) \exp \left(i m \theta_{i}\right)
$$

and similarly for the magnetic field.

A mode exists when the outgoing waves b $=\left[\mathbf{a}^{1, T} \ldots \mathbf{a}^{N_{\mathrm{c}}, T}\right]^{T}$, in partitioned vector notation, for al 
inclusions are self-consistent, that is, when the local ex pansion of Eq. (2) and the global expansion of Eq. (3) are equivalent. To relate the different terms with different origins in these expansions, we use Graf's addition theorem, which introduces change of basis matrices. This consistency
the field identity:

$$
[\mathbf{I}-\mathbf{R}(\mathbf{H}+\mathbf{S})] \mathbf{b}=\mathbf{0}
$$

where $\mathbf{R}=$ diag $\left(\mathbf{R}^{l}\right), \mathbf{H}$ is a change of a basis matrix, and where $\mathbf{R}=$ diag $\left(\mathbf{R}^{\prime}\right), \mathbf{H}$ is a change of a basis matrix, and
the $\mathbf{S}$ matrix represents the interactions between the inclund to the values of $n$ where the determinant of the matrix [I- $\mathbf{R}(\mathbf{H}+\mathbf{S})]$ vanishes, with the corresponding null-space vector $b$ characterizing the modal fields. The numerical procedure to find the modes is described by White et $a l^{5}{ }^{5}$ and Kuhlmey et al. ${ }^{6}$

\section{B. Determination of $\mathbf{R}^{\prime}$}

Calculating the reflection matrices, which relate the incdent field to the scattered field, requires that we match the field expansions valid inside and outside the inclusion. For circular inclusions this amounts to applicatio of the electromagnetic boundary conditions - the contin ity of the tangential components of the electric and mag circular and noncircular incl in cross section, respectively where the solid curves do note the dielectric interfaces. Two circles, $C$, the in scribed circle and $C_{2}$ the escribed circle, were drawn such that they touch the inside and outside surface of each in clusion, respectively. For the circular inclusion the in seribed and escribed circles are depicted separately; how ever, in reality they are coincident.

The field expansions valid on the annulus surrounding the inclusion that includes the escribed circle was give in Eq. (2). The ingeribed circle is in a uniform medium and does not contain any sources, so the field expansion has the form

$$
E_{z}^{\prime}=\sum_{m=-M}^{M} c_{m}^{E, l} J_{m}\left(k_{\perp}^{l} r_{l}\right) \exp \left(i m \theta_{l}\right)
$$

At this point we also introduce a Fourier series exparysion for each component of the electric and magnetic fields around the ith inclusion, e.g.

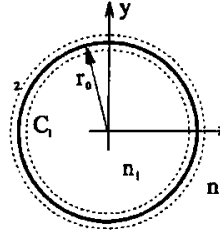

(a)

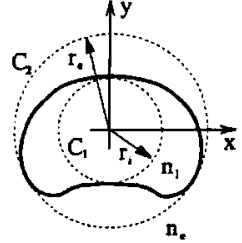

(b)
Fig. 2. Comparison between a circular and a noncircular inclusion. The circles $C_{1}$ and $C_{2}$ touch the inside and outside of each inclusion, respectively, and have radii $r_{i}$ and $r_{c}$ for the noncircelar inclusion and radius $r_{0}$ for the circular inclusion. Then has refractive index $n_{l}$ and the background $n_{e}$
clusion

$$
E_{z}^{\ell}=\sum_{m=-M}^{M} e_{z, n}(r) \exp \left(i m \theta_{l}\right)
$$

where $\varepsilon_{z, m}(r)$ is the $m$ th Fourier coefficient of the $z$ component of the electric field, with similar expansions and expansion coefficients for the other field components. We
also introduce vectors of the form $e_{z}$ of length $2 M+1$, whose $i$ th element is $e_{z, i}(r)$

For the circular inclusion the escribed and inscribed circles are coincident, and matching the field expansions requires that we impose continuity conditions on the tangential field components on the dielectric boundary. We do this by equating the Fourier coefficients of the $z$ and $\theta$ field components, where $\theta$ is the azimuthal angle. The $E_{\theta}$ and $H_{\theta}$ fleld expansions are calculated with Maxwell partitioned vector of Fourier coefficients $v^{t}(r)$ $=\mathbf{e}^{l, T} \mathbf{h}^{i, T} \mathbf{e}^{l, T} \mathbf{h}^{l, T} T^{T}$, where $T$ denotes transposition, and the matrices $\mathbf{P}^{j}$ and $\mathbf{P}_{i}^{i}$ that relate the Fourier series on the escribed and inscribed circles, respectively for the th inclusion

$$
\begin{aligned}
& \mathbf{v}^{l}\left(r_{\boldsymbol{c}}\right)=\mathbf{P}_{e}^{l}\left[\begin{array}{l}
\left.\mathbf{a}^{l}\right] \\
\left.\mathbf{b}^{l}\right]
\end{array}\right] \\
& \mathbf{v}^{l}\left(r_{b}\right)=\mathbf{P}_{i}^{l} \mathbf{c}^{l},
\end{aligned}
$$

the matching conditions become

$$
\begin{aligned}
\mathbf{v}^{l}\left(r_{e}\right) & =\mathbf{v}^{l}\left(r_{i}\right), \\
\mathbf{P}_{e}^{[}\left[\mathbf{b}^{l}\right] & =\mathbf{P}_{i}^{l} \mathbf{c}^{l} .
\end{aligned}
$$

The matrices $\mathbf{P}_{i}^{\prime}$ and $\mathbf{P}_{e}^{\prime}$ are given in Appendix A.

For noncircular inclusions the inscribed and escribed circles are not coincident; and to relate the multipole amintegration is required. An ordinary differential equation (ODE) in the Fourier coefficients of the $z$ and $\theta$ components of the fields is formed from Maxwell's equation where $O$ is a linear operator defined in Appendix B:

$$
\frac{\mathrm{d}}{\mathrm{d} r} \mathbf{v}^{\mathrm{l}}(r)=\mathbf{O v}^{\prime}(r)
$$

Because the problem is linear, integration of this ODE from the inscribed to the escribed circle yields a matrix $N$ scribed circle to the escribed circle:

$$
\mathbf{v}^{\ell}\left(r_{e}\right)=\mathbf{N}^{l} \mathbf{v}^{l}\left(r_{i}\right) .
$$
We can now equate the analytic [Eq. (7)] and numerical
[Eq. (12)] expressions for the fields on the escribed circle

$$
\mathbf{v}^{l}\left(r_{e}\right)=\mathbf{P}_{e}^{l}\left[\begin{array}{c}
\mathbf{a}^{l} \\
\mathbf{b}^{l}
\end{array}\right]=\mathbf{N}^{l} \mathbf{v}^{l}\left(r_{i}\right)=\mathbf{N}^{i} \mathbf{P}_{i}^{l} \mathbf{c}^{l}
$$

from which the reflection matrix follows when we multi ply through by $\mathbf{P}_{e} e^{\prime}-1$ and introduce two $2 \times 2$ block matrices $\mathbf{Q}_{1}$ and $\mathbf{Q}_{2}^{2}$ that are the upper and ower
of the matrix $\mathbf{P}_{e}^{i-1} \mathbf{N}^{l} \mathbf{P}_{i}^{\prime}$. Equation (13) becomes $\left[\begin{array}{l}\mathbf{a}^{l} \\ \mathbf{b}^{l}\end{array}\right]=\mathbf{P}_{e}^{l, \cdots 1} \mathbf{N}^{\prime} \mathbf{P}_{i}^{l}\left(\mathbf{c}^{l}\right)$

(14)

$=\left[\begin{array}{l}\mathbf{Q}_{1}^{l} \\ \mathbf{Q}_{2}^{l}\end{array}\right]\left(\mathbf{c}^{b}\right)$.

Eliminating the interior $\mathbf{c}_{l}$ coefficients yields the reflection matrix

$$
\mathbf{b}^{l}=\mathbf{Q}_{2}^{l} \mathbf{Q}_{1}^{l, \cdots}=\mathbf{R}^{l} \mathbf{a}^{l} .
$$

C. Numerical Solution of the Scattering Problem Here we detail the construction and solution of ODE $[\mathrm{Eq}$ (11) using a FFF method, ${ }^{, 8}$ a differential equation method originally developed for the modeling of diffraction gratings but which is applicable to any periodic boundary-value problem. Differential equation methods project Maxwell's equations onto a complex Fourier series, such that the propagation equations reduce to a set of coupled linear ordinary differential equations in the whknown expansion coefficients of the fields. Compared method achieves much faster convergence of the Fourier series for the fields. This is done through use of the factorization rules presented by $\mathrm{Li}^{15}$, which address the problem of convergence of the Fourier series for three problem of convergence of the Fourier series for three
cases of products of two periodic discontinuous functions. This problem arises in dielectrics when the product between the electric field and the refractive index squared must be expanded. For example, although both $E_{\text {nurrai }}$ and $n^{2}$, where $n$ is the refractive index, are discontinuous functions at a dielectric interface, their product is con tinuons. For discontinuous periodic functions without concurrent jump discontinuities, Laurent's rule ${ }^{1 \overline{5}}$ yields Fourier series with the best possible convergence. For is individually discontinuous but that together havi continuous product, the inverse rule ${ }^{i 5}$ is used.

\section{Foctorization Rules}

Laurent's rule is the standard technique used to expand the product of two arbitrary functions in Fourier series. For two functions $f$ and $g$, the $n$th Fourier coefficient of their product $f g$ is given by

$$
(f g)_{n}=\sum_{m=:=\infty}^{\infty} f_{n-m} g_{m}=\sum_{m=1=\infty}^{\infty} g_{n-m i} f_{m},
$$

where $f_{n}$ and $g_{n}$ are the $n$th Fourier coefficients of the functions $f$ and $g$. For an infinite number of Fourier coefficients, Eq. (17) is exact; however, its validity is not obvious when the Fourier series are truncated. For funcmather best way to factor the function product when the series are trumcated Designating $[g]$ to be the vector $[s]$ $=g_{n}$ and $[f]$ to be the Toplitz matrix whose $(n, m)$ en try is $f_{2-m}$, where $n, m \in(-M, M)$, Eq. (17) becomes

$$
[f g]=[[f]][g] .
$$

When $f$ and $g$ are individually discontinuous but have continuous product, the inverse rule ${ }^{15}$ is used:

$$
[f g]=[1 / / f]]^{-1}[g]
$$

which is obtained when we apply Laurent's rule to (1/f) $\times(f g)=g$ and invert $[[1 / f]]$. Laurent's rule can be applied because ( $f g$ ) is a continuous function at the discontinuities in $f$ and $g$ by definition, and so the product

We want to work with vectors of Fourier coefficients Whe felds so in the above examples 8 is a field component and $f$ is associated with a material property.

2. Ordinary Differentiol Equation Construction with the Fiast Fourier Factorization Method

The formulation of ODE [Eq. (11)] with the FFF method for the particular problem of scattering of light from a diby Neviere and Popov. The electric and magnetic field vectors are denoted with superscript vectors, and any symbol in bold follows the conventions of the previous sections, with bold lower-case letters representing vectors and bold capitals roprasenting matrices. The derivation url equations a dielectric:

$$
\begin{aligned}
& \nabla \times \vec{E}=i k_{0} \vec{K}, \\
& \nabla \times \vec{K}=-i k_{0}\left(n^{2} \vec{E}\right),
\end{aligned}
$$

where $\vec{K}=Z_{0} \vec{H}=\left(\mu_{0} / \epsilon_{0}\right)^{1 / 2} \vec{H}$ is a scaled magnetic field. Note the product of the refractive index squared and the electric field in Eq. (21). In component form we have six equations in the six field components, and expanding the helds and refractive index in a complex Fourier series at $r$ in an exp(imt) basis we obtain a set of $6 \times(2 M+1)$ are included. In Eq $(21)$ the function products $\left[n^{2} E_{r}\right]$, $\left[n^{2} E_{t}\right]$, nad $\left[n^{2} E_{2}\right]$ must be factorized to be expressed as functions of $\left[E_{r}\right],\left[E_{i}\right]$, and $\left[E_{z}\right]$. This is done with the

In view of factorizing the tangential and normal components of the electric field, we introduce the unit normal $\vec{N}$ to a closed curve that can be described by the function $f(r, \theta)=r-g(\theta)=0$, where $g$ is a single-valued function:

$$
\vec{N}=(\vec{v} f) /|\vec{\nabla} f| .
$$

Defining $\vec{E}_{N}=\vec{N}(\vec{N} \cdot \vec{E})_{3}$, the normal component of the electric field, and $\vec{E}_{T}=\vec{E}-\vec{E}_{N}$, the tangential component of the electric field, we can express $\left[n^{2} \tilde{E}\right]$ as

$$
\left[n^{2} \vec{E}\right]=\left[n^{2} \vec{E}_{T}+n^{2} \vec{E}_{N}\right] .
$$

The product $n^{2} \vec{E}_{T}$ is between a discontinuous and con tinuous function, so we apply the first factorization rule, Laurent's rule. The product $n^{2} E_{N}$ is between two disconthinusus functions that have a continuous product, so we apply the inverse rule. Equation (23) becomes

$$
\left[n^{2} \vec{E}\right]=\left[\left[n^{2}\right]\right]\left[\vec{E}_{T}\right]+\left[\left[\frac{1}{n^{2}}\right]\right]^{-1}\left[\vec{E}_{N}\right]
$$


1924 J. Opt. Soc. Am. B/Vol. 21, No. 11/Novernber 2004

Substituting for the tangential and normal components of the fields and introducing a matrix $\boldsymbol{Q}_{n}$, whose elements are given in Appendix B, we obtain

$$
\left[n^{2} \vec{E}\right]=\mathbf{Q}_{n,[}[\vec{E}]=\left[\begin{array}{ccc}
\mathbf{Q}_{n^{2},, r r} & \mathbf{Q}_{r^{2}, r \theta} & 0 \\
\mathbf{Q}_{n^{2}, \theta r} & \mathbf{Q}_{n^{2}, \theta \theta} & 0 \\
0 & 0 & \mathbf{Q}_{n^{2}, z z}
\end{array}\right][\vec{E}] .
$$

We can now obtain expressions for $\left[E_{r}\right],\left[E_{f}\right]$, and $\left[E_{z}\right]$, although here we show only the result for $\left[E_{r}\right]$. From Eq. (25) we can see that

$$
\left.\left[n^{2} E_{r}\right] \ldots \mathbf{Q}_{n^{2}, \ldots r}\left[E_{r}\right]+\mathbf{Q}_{n^{2}, \ldots}, E_{\theta}\right],
$$

and so usin

$$
i \alpha_{\theta} \frac{\left[K_{z}\right]}{r}-i \beta\left[K_{\theta}\right]=-i h_{\theta}\left[n^{2} E_{r}\right]
$$

from the second Maxwell curl equation, we obtain

$$
\begin{aligned}
{\left[E_{r}\right]=} & \mathbf{Q}_{n^{2}, r r}{ }^{1}\left\{\left[n^{2} E_{r}\right]-\mathbf{Q}_{n^{2}, r+r}\left[E_{f]}\right]\right\}, \\
= & \mathbf{Q}_{n^{2}, r r}\left\{\frac{\beta\left[K_{\theta]}\right]-\frac{1}{r} \mathbf{M} \alpha_{d}\left[K_{2}\right]}{k_{0}}\right. \\
& \left.-\mathbf{Q}_{n^{2}, r r}\left[E_{\theta}\right]\right\} .
\end{aligned}
$$

We can similarly obtain expressions for $\left[E_{\nu}\right]$ and $\left[E_{z}\right]$.

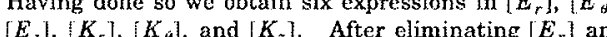
$\left[K_{z}\right],\left[K_{r}\right],\left[K_{, j}\right]$, we are lett with four expressions in $\left.\left[E_{0}\right],\left[E_{z}\right], K_{i}\right]$ and $\left[K_{z}\right]$ that involve only derivatives with respect to $r$

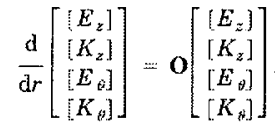

or in the notation of Subsection 2.B,

$$
\frac{\mathrm{d}}{\mathrm{d} r} \mathbf{v}^{l}(r)=\mathbf{O v}^{l}(r),
$$

where the coefficient matrix $O$ is given in Appendix $B$. if $j=i$ and zero otherwise, yields the matrix $\mathbf{N}^{l}$ introduced in $E_{q}(12)$ Once $\mathbf{N}^{l}$ has culated the reftection matrix $\mathbf{z}^{l}$ in Eq (16) can be calcucuted when we apply the matehing conditions in Eq. (13). If $L$ is factorization rules had not been appliod, the matrix $\mathbf{Q}_{n^{2}}$ would have been a diagonal matrix in the Toeplitz matrix for the refractive index squared $\left[\left[n^{2}\right]\right]$ and the system of equations obtained would be much sim pler. However, as shown by Nevière and Popov, ${ }^{9}$ the convergence is significantly improved when Li's factorization rules are used

\section{NUMERICAL IMPLEMENTATION AND}

A. Numerical Proced

A ForTran code was developed and combined with an existing implementation of the multipole method. "When Toeplitn matrices for the mompents of the

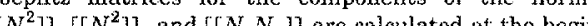
ning of the integration and used throughout. The Toeplitz matrices for the refractive index $\left[\left[n^{2}\right]\right]$ and $\left.\left[1 / n^{2}\right]\right]$ are calculated at each step, and no angular discretization is necessary when the refractive index is piecewise constant. In this case the Toeplitz matrice can be calculated exactly if the refractive-index profile is known analytically. A fourth-order Runge Kutta algorithm is used to integrate the ODE $\mid$ Eq. (30). Applying the matching conditions yields the reflection matrix for the inclusion. The refection matrices need to be calenlated only for inclusions with a unique geometry, and simple change of basis operation can be used to find the reffection matrix
ent orientation

\section{B. Convergence}

The convergence of the modal effective indices $n_{\text {eff }}$ in this implementation depends on the number of multipoles $M$ retained in forming the field identity and the numerical errors introduced into the reflection matrices. As $M$ increases, the agreement between the local expansions and the global expansions improves until the conditioning of the matrices entering the field identity limits the agree ment. The numerical differences between the expancation of truncation errors-both the errors introduced when $M$ is too small and the errors due to conditionin wis large To characterize the agroement between local and global expansions, Kuhlmey et $a l^{6}{ }^{6}$ introduced

$$
W=\frac{\int_{C_{z}}\left|E_{z}^{\text {local }}\left(\theta_{l}\right)-E_{z}^{\text {Wijngatard }}\left(\theta_{l}\right)\right| \mathrm{d} \theta_{l}}{\int_{C_{2}}\left|E_{z}{ }^{\text {Wijgzarai }}\left(\theta_{l}\right)\right| \mathrm{d} \theta_{l}},
$$

which is evaluated on the escribed circle of the $l$ th inclu-

The convergence of the local expansions depends on the
The number of multipoles. For circular inclusions, the interaction can be represented with relatively few multipole For more complex inclusion geometries, the fields scattered by the inclusion have larger angular variations, so field. With increasing $M$ W decreases and the conver

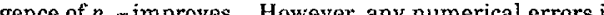

\begin{tabular}{|c|c|c|c|c|}
\hline$M_{\operatorname{minx}}$ & $\operatorname{Re}\left(n_{\mathrm{en}}\right)$ & $\operatorname{Im}\left(n_{\mathrm{eff}}\right) \times 10^{-6}$ & $W_{d x t}$ & $W_{\text {dasti }}$ \\
\hline 4 & 1.446419485 & 2.9437 & $3.4 \times 10^{-3}$ & $5.5 \times 10^{-3}$ \\
\hline 5 & 1.446411348 & 1.4287 & $20 \times 10^{-3}$ & $4.2 \times 10^{-3}$ \\
\hline 6 & 1.446397187 & 2.1808 & $2.7 \times 10^{-4}$ & $1.7 \times 10^{-3}$ \\
\hline 7 & 1.446396099 & 2.4601 & $1.2 \times 10^{-1 .}$ & $5.4 \times 10^{-1}$ \\
\hline 8 & 1.446397463 & 2.3382 & $1.1 \times 10^{0.4}$ & $3.5 \times 10^{-4}$ \\
\hline 9 & 1.446397587 & 2.3116 & $4.7 \times 10^{-2}$ & $1.8 \times 10^{-4}$ \\
\hline
\end{tabular}
the reflection matrix cause the effective index to converge curately is critical. Increasing the spatial resolution in the integration, which is determined only by the number of radial integration steps when the refractive-index profile is known analytically, improves the convergence. The convergence can be further improved when higherorder Fourier coefficients are retained during the reflec
Table 1. Convergence of $n_{\text {eff }}$ with $M^{\text {a }}$

${ }^{\circ}$ Kesults are for the polarization of the fundanentul mode with the

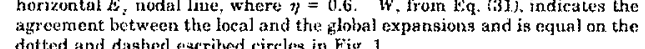

tion matrix calculation, which are discarded when the field identity is formed. This is because in general there is coupling between different arders in the form of eonvolutions, so performing the numerical integration with extra Fourier coeficlents improves the convergence of the modal effective indices.

In the followine the MOF considered (shown in Fis 1$)$ has identical inclusions with fixed semimajor axis a $-25 \mu \mathrm{m}$ and varying semiminor axis $b=0$, with $a$ inclusion separated by a center-to-center distance of $A$ $=6.75 \mu \mathrm{m}$. The ellipticity of the inclusions is defined as
$=6.10$ the ratio of the minor to the major axis and is given by $\eta$. All modes are calculated at the wavelength $\lambda$ $=1.45 \mu \mathrm{m}$. The inclusions have refractive index $n_{t}$ 1 , and the brackground refractive index is $n_{e}=1.45$.
A jacket with refractive index $n_{j}=1.45+2.9 \times 10^{\circ}$ A jacket with refractive index $n_{j}=1.45+2.9 \times 10^{-13}$
was included in the calculation s; however the jacket was was included in the calculations; however, the jacket was found to have no effect on the modal effective indices
within the precision presented in this subsection. The modes of the equivalent structure with circular inclusions are either singly or doubly degenerate. For $\eta<1$, all dereneracies are lifted so here we follow the two perpendicular polarizations of the fundamental modes of the circular structure as $\eta$ is varied.

Table 1 shows the convergence of the effective index of the $E_{z}$ polarization with a horizontal nodal line of the fundamental mode as the number of multipoles is varied The number of multipoles used in the calculation of the reflection matrix is $M=10$, and $\eta$ is fixed at 0.6. In cluded in Table 1 is the $W$ parameter, which indicates the agreement between the local and the global expansions. Because of the modal symmeties, $W$ is equal on the dashed and dotted escribed circles in Fig. 1. The rea part of the effective index can be seen to converge to seven significant figures and the imaginary part converges to 2 As the number of multipoles is increased, $W$ decreases the conditioning of the matrices that onter the field identity. If the inclusions were circular, the convergence for $M$ $=9$ is to ten and four significant figures in the real and imaginary parts, respectively, because of the smaller angular variations in the fields scattered by a circular inclusion and the fact that the reflection matrices are know analytically.

A direct comparison was made with the $\mathrm{ABC}$ method for the MOF considered here. The effective indices of the
Campbell et $a$ d.

two polarizations of the fundamental mode were calcuated as a function of $\eta$. The effective indices of the different polarizations of the fundamental mode as calcu presented in Tables 2 and 3 . For the DMM, $M-9$ is used to form the field identity, and $M=1.0$ is used to cal culate the reflection matrix. Sufficient integration steps were taken such that the convergence was multipole limited. For the $\mathrm{ABC}$ model, the radial resolution, which refers to the number of points in the radial direction at which the fields are calculated, was 1000; and the angular resolution, which refers to the truncation of the Fourier series, was 60 . Both the ABC results and the DMM results have converged to seven significant figures in the real part of the effective index and converged to two in the maginary part, ${ }^{16}$ which is in agreement with one results. nificant figures in $\operatorname{Re}\left(n_{\mathrm{ef}}\right)$ and one in Im(n $\left.n_{\mathrm{eff}}\right)$, although

Table 2. Comparison between the DMM and the ABC Method for the Polarization of the Funda-

mental Mode with the Forizontal $E_{z}$ Nodal Line

1

DMM 1.44396232

$\begin{array}{lll}\text { DMM } & 1.445395232 & 3.1945 \times 10^{-8} \\ \text { ABC } & 1.445397228 & 3.2376 \times 10^{-8} \\ 0.9 & 1.45672782 & 1.1090 \times 10^{-8}\end{array}$

0.8

DMM

$\mathrm{ABC}$

0.8

$\mathrm{ABC}$

$\mathrm{DMM}$
$\mathrm{ABC}$
0.6

$0.6 \mathrm{DMN}$

$\mathrm{ABC}$

$\begin{aligned} & 1.445672782 \\ & 1.445672267\end{aligned} \quad 1.1040 \times 10^{-7}$

$3.5723 \times 10^{-1}$

$1.445929388-3.5723 \times 10^{-7}$

$1.446169785 \quad 9.8458 \times 10^{-17}$

$\begin{array}{ll}1.446397587 & 2.311 .6 \times 10^{-6} \\ 1.446404071 & 29339 \times 10^{-6}\end{array}$

Table 3. Comparison between the DMM and the ABC Method for the Polarization of the Fundamental Mode with the Vertical $E_{\approx}$ Nodal Lin

\begin{tabular}{lll}
\hline$\eta$ & \multicolumn{1}{c}{$\operatorname{Re}\left(n_{\text {eff }}\right)$} & \multicolumn{1}{c}{$\operatorname{Iru}\left(n_{\text {eff }}\right)$} \\
\hline 1 DMM & 1.445395232 & $3.1945 \times 10^{-8}$ \\
ABC & 1.445397228 & $3.2376 \times 10^{-8}$ \\
0.9 & & $1.44560 \times 10^{-7}$ \\
DMM & 1.45677647 & $1.3560 \times 10^{-7}$ \\
ABC & 1.445676964 & $1.3624 \times 1$ \\
0.8 & 1.445941057 & $4.6190 \times 10^{-7.7}$ \\
DMM & 1.445941003 & $4.8042 \times 10^{-7}$ \\
ABC & & \\
0.7 & 1.446189882 & $1.2755 \times 10^{-6}$ \\
DMM & 1.4461916 & $1.4230 \times 10^{-6}$ \\
ABC & & \\
0.6 & 1.446427235 & $2.9601 \times 10^{-6}$ \\
DMM & 1.44643281 & $3.6624 \times 10^{-6}$ \\
ABC & & \\
\hline
\end{tabular}


this is less than the internal convergence of each method. The reason for this is not presently clear and will be the subject of future research. Each individual calculation in Tables 2 and 3 for the DMM required approximately 8 megabytes of memory used. Similar calculations to those above for circular inclusions would take approximately $5 \mathrm{~s}$ and use a similar amount of memory. Thus the DMM is an efficient technique to model MOFs in a destop envian efficient technique to mocel
ronment when high accuracy is required. The computational cost of calculating the reflection matrices numerically dominates that of forming and calculating the eigenvalues and eigenvectors of the field identity for small numbers of identical inclusions. However, when the number of inclusions $N_{c}$ ws 25 , the computational cost of calculating the eigenvectors of the field identity begin to become significan, and for larger numbers of inclusions, the main computational overhead is the solution of the field identity for the modal effective indices.

\section{INCLUSION MICROSTRUCTURED OPTICAL}

For an application of the DMM, we consider a MOF with liptical inclusions previously studied by Steel and Osgood cosing the plane-wave method. Our motiva-
tion for choosing this fiber is to answer an unresolved tion for choosing this fiber is to answer an unresolved
question regarding the cutoffs of the two polarizations of the fundamental mode. One useful picture of modal cutoffs involves the fundamental space-filling mode (FSM), which in a step-index circular fiber is the radiation mode with the highest effective index. In a MOF geometry the FSM states are the fundamental states of the infinite photonic crystal cladding if the core is absent. In this case a mode is guided in the core of the fiber if the real part $\beta^{r}$ of the propagation constant of the mode is greater than the wave number in the core:

$$
k_{0} n_{\text {corr }}>\beta^{r}>\beta_{\mathrm{rSM}} .
$$

The effective index $n_{\text {eff }}=\beta_{\text {rSM }} / k_{n}$ of the FSM can be thought of as the effective cladding index of the fiber. In a MOF with elliptical inclusions with the same oriFor certain geometries the fundamental mode, split propagation constant of one of the fundamental mode could pass through the FSM with the opposite polarization. They proposed that the mode would become cut of at this point, but were unable to answer this question definitively because of the inability of the plane-wavo method to calculate confinement loss. Subsequent modeling by beam propagation was unable to resolve the issuce becausy of the lange lossos near the modal cutoffs. If the mode did become cut off, the fiber would support only gle polarization of the fundamental mode.

The structure modeled here, inset in Fig. 3, consists of seven rings of elliptical inclusions arranged on a hexagoinclusion has a major axis of 2.8546 m and a minor axis of $1.4273 \mu \mathrm{m}$ so $b=0.5 a$ The inclusion refrative in dex is $n_{b}=1$, the background refractive index is $n$
$=1.45$, and a jacket with refractive index $n_{j}=1.45$ $+2.9 \times 10^{* 4}$ was included. The jacket was found to have no effect on the modal effective indices. The fabrication of a MOF with elliptical inclusions in which the majis hole pitch was $3.82 \mathrm{\mu m}$, the inclusions had a major axis of $2.1 \mu \mathrm{m}$, and the ratio of major to minor axis was modeled here is a realistic design. In the modeling, $M$ - 9 multipoles is ured in the calculation of the reflection matrices and $M=7$ is used in the formation of the field identity. In Fig. 3 the real parts of the FSMs and the fundamental modes' effective indices are plotted as a function of the dimensioniess frequency $y \cdots \cdots, \omega /(2 \pi c)$ for consistency with Stecl and Osgood. ${ }^{17,1 \mathrm{H}}$ The frequency in hertz is given by $f=c w / \Lambda$.

At $z^{\prime}=0.7$, the polarization of the fundamental mode with the horizontal $E_{z}$ nodal line (guided $x$ ) passe through the FSM with the vertical $E_{z}$ nodal line (FSM $y$ If the mode was to become cut off for lower frequencies, we would expect its confinement loss to increase dramatically for 4.7 . Higure 4 is a plot of the imaginary part damental mode. The confinement lous in decibels per kilometer is given by $\mathrm{E}$ (1) We can see that the loss the guided $x$ mode starts to increase dramatically for $<0.9$; however, in contrast to the prediction made by Steel and Osgood, the logs curve of the guided $x$ mode $i$ unaffected by the crossing of the FSM $y$ state at $y \ldots 0$. Furthermore, the loss of the guided $x$ mode is actually smaller than that of the guided $y$ mode. This is because

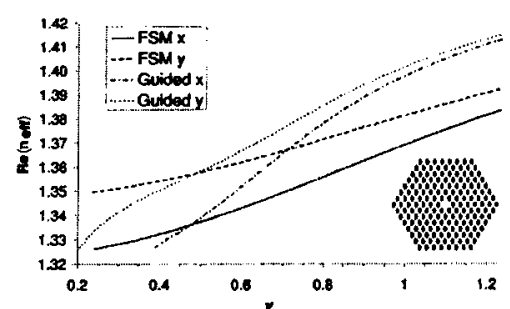

Fig. 3. Real part of the effective index of the fundamental modes and the FSMs of a seven-ring hexagonal MOF (inset) with
elliptical inclusions oriented vertically with the major axis.

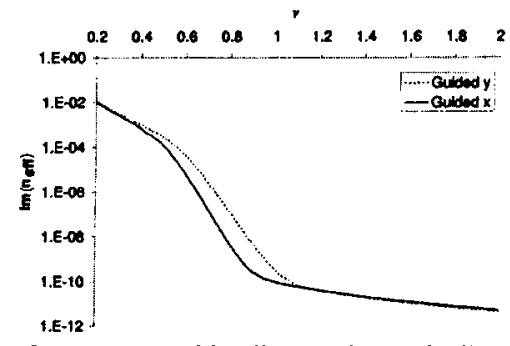

Fig. 4. Imaginary part of the effective index on a log-linear scale
of the fundamental modes of a seven-ring hexagonal MOF with elliptical inclusions oriented vertically with the major axis. A $v=0.65$ the ration
$y$ mode is 0.02 the difference in the effective index of the guided $x$ mode and the FSM $x$ state is larger than the difference between the guided $y$ mode and the FSM $y$ state. In the effective cladding picture the mode with effective index closest to the corresponding effective cladding index would have tho More generally Fig, 4 indicates three different trend in the modal losses. For frequencies $y>11$ the confins ment loss is small, and both modes have similar propagation constants. In this recime the modes are tightly confined to the core of the fiber. For frequencies which from Yig. 3 is the frequency at which each mode crosses its corresponding FSM, the confinement losses are large, and both modes are poorly confined. This agrees well with the effective index model for the modal cutoffs. $^{20}$ For frequencies $0.45<\nu<1.1$, we can sec a transition region where the confinement loss is changing rapidly, and the loss curves for each mode have the same shape. However, because of their relative shift there is a strong polarization-dependent loss. These two asymptotic regions and the transition region closely resemble the prediction circular inclusion

$v, \ldots .83$ the ratio of the confinement losses reaches a maximum of 27 , and the confinement losses for the two sugrest that the fiber would support only a single polar ization of the fundamental mode at the wavelength of $A$ .. $4769 \mathrm{~nm}$. Kubota et al..$^{22}$ have reported a MOF where the loss ratio between the two polarizations of the fundamental mode is of the order of 8 at $a$ wavelength of $\lambda$ $=1550 \mathrm{~nm}$. Measurements indicated that the fiber sup ported only a single polarization of the fundamental mode. It is likely that a MOF consisting of a hexagonal array of elliptical inchusions could be designed to similar specifications at a wavelength of $1550 \mathrm{~nm}$; however. thi question is beyond the scope of this paper.

\section{DISCUSSION AND CONCLUSIONS}

In this paper we have extended an efficient existing method for modeling MOFs with the multipole method to fiber geometries with noncircular inclusions through use local expansions about the arbitrary inclusions. The method was shown to be in excellent agreement with an existing method. Applying the method to a fiber with seven rings of elliptical inclusions revealed that the fiber effectively supported a single polarization of the fundamental mode.

One of the features of the FFF method is that it does not lose accuracy with increasing refractive-index contrast. Thus our method will retain its accuracy for chatcogenide and other high-index MOFs under investigation. The refiactive indices can atho be complex, on materia The formulation bere

列

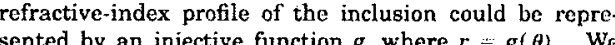
could overcome this restriction by representing the refractive-index profile as a number of sinply connected domains, and inclusions with arbitrary genmetry could hen be studied.

Finally, more general formulations of the FFF method are applicable to anisotropic media. For example, MOFs could be studied.

\section{APPENDIX A: REFLECTION MATRIX}

We consider an arbitrary inclusion as shown in Fig. 2 We use the Fourier series expansions introduced in $\mathbf{E q}$. nents. To relate the multipole amplitudes to the Fourier coefficients, we must calculate the $\theta$ feld components using Maxwell's equations ${ }^{10}$ :

$$
\begin{aligned}
& E_{\theta}(r, \theta)=\frac{i}{k_{\perp}^{2}}\left(\frac{\beta}{r} \frac{\partial E_{z}}{\partial \theta}-\cdots k_{0} \frac{\partial K_{z}}{\partial r}\right), \\
& K_{p}(r, \theta)=\frac{i}{k_{\perp}^{2}}\left(\frac{\beta}{r} \frac{\partial K_{z}}{\partial \theta}+k_{0} n^{2} \frac{\partial E_{2}}{\partial r}\right),
\end{aligned}
$$

where $n$ is the appropriate refractive index

Calculating the $E_{\theta}$, and $K_{k}$ field components and equating the Fourier series and multipol
the inscribed circle yields the matrix $\mathbf{P}_{:}^{\prime}$

$$
\left[\begin{array}{l}
\mathbf{e}_{2}^{l}\left(r_{i}\right) \\
\mathbf{k}_{z}^{l}\left(r_{i}\right) \\
\mathbf{e}_{\beta}^{l}\left(r_{i}\right) \\
\mathbf{k}_{\beta}^{l}\left(r_{i}\right)
\end{array}\right]=\mathbf{P}_{i}^{l}\left[\begin{array}{c}
\mathbf{c}^{E, j} \\
\mathbf{e}^{K, l}
\end{array}\right] .
$$

where

$$
\mathbf{P}_{i}^{\prime}=\left[\begin{array}{cc}
\mathbf{J}_{i} & \mathbf{0} \\
\mathbf{0} & \mathbf{J}_{i} \\
-\frac{\beta}{k_{L}^{i .2} r_{i}} \mathbf{M J}_{i} & -\frac{i k_{0}}{k_{\perp}^{i}} \mathbf{J}_{i}^{\prime} \\
\frac{i k_{\mathrm{o}} n_{i}^{2}}{k_{\perp}^{i}} \mathbf{J}_{i}^{\prime} & \frac{\beta}{k_{L}^{i, 2} r_{i}} \mathbf{M J}_{i}
\end{array}\right],
$$

and $\mathbf{M} \ldots \operatorname{diag}(m), \quad \mathbf{J}_{i} \ldots \operatorname{diag}\left(J_{m i}\left(k_{i}^{i} r_{i}\right)\right]$, and $\mathbf{H}_{i}$ The primes denote derivatives with respect $J_{i}$ and $\mathbf{H}_{i}$ ment.

Repeating this on the escribed circle yields $\mathbf{P}_{e}$ ?

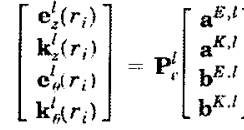




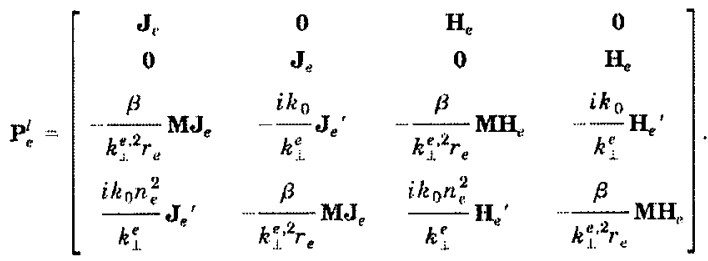

The last element required is the matrix $\mathbf{N}^{l}$, which represents the numerical integration from the inscribed to the escribed circles. Equating the analytic and numercal expressions for the fields on the escribed circle, we obtain Eq. (13). Multiplying through by $\mathbf{P}_{*}$ and

$$
\left[\begin{array}{l}
\mathbf{a}^{E, l} \\
\mathbf{a}^{K, l} \\
\mathbf{b}^{E, l} \\
\mathbf{b}^{K, l}
\end{array}\right]=\mathbf{P}_{e}^{-1} \mathbf{N}^{i} \mathbf{P}_{i}\left[\begin{array}{c}
\mathbf{c}^{E, l} \\
\mathbf{c}^{K, l}
\end{array}\right]=\left[\begin{array}{l}
\mathbf{Q}_{1} \\
\mathbf{Q}_{2}
\end{array}\right]\left[\begin{array}{l}
\mathbf{c}^{E, l} \\
\mathbf{c}^{K, l}
\end{array}\right],
$$

allows us to eliminate the interior coefficients $\mathbf{C}^{l}$ to form the reflection matrix

$$
\left[\begin{array}{l}
\mathbf{R}^{E} \\
\mathbf{B}^{K}
\end{array}\right]=\mathbf{Q}_{2} \mathbf{Q}_{1}{ }^{l}\left[\begin{array}{l}
\mathbf{A}^{E} \\
\mathbf{A}^{K}
\end{array}\right]=\mathbf{R}^{l}\left[\begin{array}{l}
\mathbf{A}^{E} \\
\mathbf{A}^{K}
\end{array}\right] .
$$

\section{APPENDIX B: ORDINARY DIFFERENTIAL}

\section{NQUATICA}

The matrix $\mathbf{Q}_{n^{2}}$ follows from Eq. (24), and use of $\left[N_{n}^{2}\right]$ $+\left[\left[N_{r}^{2}\right]\right]=\mathbf{I}$, where $\mathbf{I}$ is the identity matrix that follow from the normal being a unit vector. $\mathbf{Q}_{n}$ " then has the

$$
\mathbf{Q}_{n^{2}}=\left[\begin{array}{ccc}
\mathbf{Q}_{n^{2}, r r} & \mathbf{Q}_{n^{2}, r^{* i l}} & \mathbf{0} \\
\mathbf{Q}_{n^{2}, t r r} & \mathbf{Q}_{n^{2}, \boldsymbol{\rho} \theta} & \mathbf{0} \\
\mathbf{0} & \mathbf{0} & \mathbf{Q}_{n^{2}, z z}
\end{array}\right],
$$

where

$$
\begin{aligned}
& \mathbf{Q}_{n^{2}, r r}=\left[[ n ^ { 2 } ] \left[\left[\left[N_{\theta}^{2}\right]\right]+\left[\left[\frac{1}{n^{2}}\right]\right]^{-1}\left[\left[N_{r}^{z}\right]\right],\right.\right. \\
& \mathbf{Q}_{n^{2}, r u}=-\left(\left[\left[n^{2}\right]\right]-\left[\left[\frac{1}{n^{2}}\right]\right]^{-1}\right)\left[\left[N_{r} N_{\theta}\right]\right], \\
& \mathbf{Q}_{n^{2}, \theta r}=-\left(\left[\left[n^{2}\right]\right]-\left[\left[\frac{1}{n^{2}}\right]\right]^{-1}\right)\left[\left[N_{r} N_{f}\right]\right], \\
& \mathbf{Q}_{n^{2}, \theta \psi}=\left[\left[n^{2}\right]\right]\left[\left[N_{r}^{2}\right]\right]+\left[\left[\frac{1}{n^{2}}\right]\right]^{1-1}\left[\left[N_{\theta}^{2}\right]\right], \\
& \mathbf{Q}_{n^{2}, z z}=\left[\left[n^{2}\right]\right] .
\end{aligned}
$$

Without Li's factorization rules, $\left[\left[1 / n^{2}\right]\right]^{-1}$ would be replaced with $\left[\left[n^{2}\right]\right]$ and $\mathbf{Q}_{n^{2}}$ would be a diagonal matrix in
$O_{11}=0$,

$\mathbf{O}_{12}=-\frac{i \beta}{r k_{n}} \mathbf{Q}_{n^{2}, r}{ }^{1}, \mathbf{M}$,

$\mathbf{O}_{13}=-i \beta \mathbf{Q}_{n^{2}, r r}^{1} \mathbf{Q}_{n^{2}, r \theta}$,

$\mathbf{O}_{14}=\frac{i \beta^{2}}{k_{0}} \mathbf{Q}_{n^{2}, r r}^{\frac{1}{2}}-i k_{0} \mathbf{I}$,

$\mathbf{O}_{21}=\frac{i \beta}{r k_{0}} \mathbf{M}$

$\mathbf{O}_{22}=\frac{i}{r} \mathbf{Q}_{n^{2}, \theta r} \mathbf{Q}_{n^{2}, r, r} \mathbf{M}$,

$\mathbf{O}_{23}=-\frac{i \beta^{2}}{k_{0}} \mathbf{I}+i k_{0}\left(\mathbf{Q}_{n^{3}, \theta r}-\mathbf{Q}_{n^{2}, \theta r} \mathbf{Q}_{n^{2}, r r} \mathbf{Q}_{n^{2}, r \theta}\right)$,

$\mathbf{O}_{24}=i \beta \mathbf{Q}_{n^{2}, \theta r} \mathbf{Q}_{n^{2}, r r}^{1}$

$\mathbf{O}_{31}=\mathbf{0}$,

$\mathbf{O}_{32}=i k_{0} \mathbf{I}-\frac{i}{r^{2} k_{0}} \mathbf{M Q} \mathbf{Q}^{2}, r, \mathbf{M}$,

$\mathbf{O}_{33}=-\frac{1}{r} \mathbf{I}-\frac{i}{r} \mathbf{M Q}_{n^{2}, . r}^{1} \mathbf{Q}_{n^{2}, r,}$,

$\mathbf{O}_{34}=\frac{i \beta}{k_{0} r} \mathbf{M Q}_{n^{2}, r r}^{1}$

$\mathbf{O}_{41}=\frac{i}{r^{2} k_{0}} \mathbf{M}^{2}-i k_{0} \mathbf{Q}_{M^{2}, z z}$,

$\mathbf{O}_{42}=\mathbf{0}$,

$\mathbf{O}_{43}=-\frac{i \beta}{r k_{0}} \mathbf{M}$,

$\mathbf{O}_{44}=\frac{1}{r} \mathbf{I}$.

\section{ACKNOWLEDGMENTS}

This research was produced with the assistance of the Australian Research Council (ARC) under the ARC Cenbandwidth Devices for Optical Systems is an ARC Centre of Excellence.
The original multipole code was provided by Bori Kuhlmey, who also developed extensions required for noncircular inclusions.

The authors thank Nader Issa for providing the $A B C$ calculations that were used in this paper and Philippe Boyer for providing in-plane seattering results that were used in the initial validation of the implementation. The authors are also grateful to Mike Steel for useful discussions and Michel Neviere and Evgeny Popov for their invergence on multipole order.

\section{REFERENCES}

1. d. K. Ranka, R. S. Windeler, and A. J. Stentz. "Optical properties of high-delta air-silica im
Opt. Lett. 25, 796-798 (2000).

. S. Windeler, and G. L Burdge, "Cladding-mode Kerbage, in air-silica microstructured optical fibres." J. Lightwave Technol. 18, 1084-1100 (2000).

eck, "Computation of mode eigencunctions in graded-index optical fibers by the p)
beam method, Appl. Opt. 19, 2240 2246 (1980)

4. N.A. lsss and L. Poladian, "Vector wave expansion method , I. Light

T. P. White, B. T. Kuhlmey, R. C. McPhedran, D. Maystre, G. Renversez, C. Martijn de Sterke, and L. C. Botten, "Mutipole method for microstructured optical fibers. I. For

6. B. T. Kuhlmey, T. P. White, G. Renverseg, D. Maystre, I. C Botten, C. Martijn de Sterke, and R. C. McPhedran, "Mul-

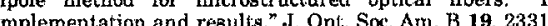
$2340(2002)$.

7. E. Popov and M. Nevière, "Maxwell equations in Fourier pace: a fast-converging formulation for diffraction by arAm. B 18, 2886-2894 (2001)
8. M. Nevière and E. Popov, Light Propagation in Periodic Me. dia (Marcel Dekker, New York, 2003). T. A. lssa, M. A. van Eijkelenborg. M. Fellow, F. Cox. G.
Henry, and M. C. J. Large. Fabrication and study of microstructured optical fibers with elliptical holes," Opt. Lett. 29. structured optical fibs

10. A. W. Snyder and J. D. Love. Optical Waveguide Theory (Chapman \& Hall, London, 1983). "Gided normal modes of two parthel cire K. M. Lo. R. C. MePhedran, I. M. Bassett, and G. W. Milton "An electronagnetic theory of dielectric waveguides with multiple embed.
$396-410$ (1994). expansion method for multiple-optical-fiber syytern," d. Lightwrave lechnol. 12, 415-417 (1994)

ridbook of Mathemati. L. Li, "Use of Fourier series in the analysis of discontinuous periodic structures," J. Opt. Sac Am. A 13, 1870-187 N. Isso

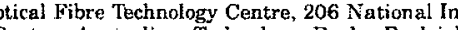
novation Centre, Australian Tlechnology Park, Eveleigh
New South Wales 1430 . Australia, n.issa@oftc.usydedu.au M.J. Stoel and B. M. Osyond, Jr., "Elliptical-hole photonic crystal fibers," Opt. Lett. 26, 229-231 (2001).

M. J. Steol and M. M. Osgood, Jr., Polarization and dlisperLightwave Technol. 19. 495-503 (2001). T. A. Birks. J. C. Knight, and P. St. J. Russell, "Endless siogle-mode photonic crystal fiber," Opt. Lett. 22, 961 96. Jich

Knight, T. A. Birks, and P. St. J. Russell, "Properties of Soc. Amic crystal fiber and the effective index nodel, $J$. Opt B. T. Kuhlmey, R. C. McPhedran, C. Martijn de Sterke, and O. A. Pobinson, "Microstructured optical fibers: where
the edge?" Opt. Express 10, 1285-1290 (2002), http: WWW.opticsexpresB.org. Kubota, S. Kawanishi, S. Koyanagi, M. Tanaka. and S Yamaguchi. "Absolutely single polarization photonic crystal
fiber," IFEF Photonics Technol. Iett. 16, $182 \cdots 184$ (2004). 